\title{
Herschel Observations of the HR 8799 Disk
}

\section{Brenda C. Matthews ${ }^{1,2}$, Grant Kennedy ${ }^{3}$, Bruce Sibthorpe ${ }^{4}$, Mark Booth $^{2,1}$, Hannah Broekhoven-Fiene ${ }^{2,1}$, Mark Wyatt ${ }^{3}$, Bruce Macintosh $^{5}$ and Christian Marois ${ }^{1}$}

\author{
${ }^{1}$ National Research Council of Canada, Herzberg Astronomy \& Astrophysics Program, 5071 \\ West Saanich Road, Victoria, BC, V9E E7, Canada, email: brenda.matthews@nrc-cnrc.gc.ca \\ ${ }^{2}$ Dept. of Physics \& Astronomy, University of Victoria, Elliott Building, 3800 Finnerty Rd, \\ Victoria, BC, V8P 5C2 Canada \\ ${ }^{3}$ Institute of Astronomy, Madingley Rd, Cambridge CB3 0HA, UK \\ ${ }^{4}$ SRON Netherlands Institute for Space Research, Groningen, The Netherlands \\ ${ }^{5}$ Lawrence Livermore National Labs
}

\begin{abstract}
The HR 8799 four-planet host is known to host a multi-component disk from Spitzer observations. We have obtained Herschel $\dagger$ observations of the disk which provide increased sensitivity and resolution of its outer components: the planetesimal belt and halo. We find that the two components cannot be discerned from the spectral energy distribution alone, but require resolved images to independently identify them. In the resolved images, the halo stands out for its steep radial profile and large radial extent to $2000 \mathrm{AU}$, a factor of two larger than was estimated from Spitzer data.
\end{abstract}

Keywords. stars: individual (HR 8799), stars: circumstellar matter, planetary systems

\section{Background}

The excess emission indicating a debris disk was first detected around the A star HR 8799 by IRAS (Moshir et al. 1990). The disk was not resolved until after the detection of a directly imaged planetary system around HR 8799 (Marois et al. 2008). Using Spitzer, $\mathrm{Su}$ et al. (2009) identified three components present in the disk: a warm inner component and two colder outer components: the planetesimal belt extending from 100-300 AU and the halo extending to 1000 AU. Excepting that very extended halo, the architecture of the HR 8799 system is very similar to that of the Solar System, with a warm inner disk, four gas giants (Marois et al. 2010) and then an outer, cold planetesimal disk.

\section{Herschel Observations}

We have obtained deep maps of HR 8799 with Herschel using both PACS (Poglitsch et al. 2010) and SPIRE (Griffin et al. 2010) and thereby obtain flux measurements for the disk at $70,100,160,250,350$ and $500 \mu \mathrm{m}$. The disk is resolved from $70-250 \mu \mathrm{m}$ (Matthews et al. 2013). Figure 1 combines the Herschel data with earlier data from Moshir et al. (1990), Williams \& Andrews (2006), Su et al. (2009), Ishihara et al. (2010) and Patience et al. (2011) to produce a well-sampled SED. We find a best fit model of two temperature components: $153 \pm 15 \mathrm{~K}$ and $36 \pm 1 \mathrm{~K}$. The Herschel data are not sensitive to the warmer component, which is traced primarily by Spitzer MIPS and IRS data ( $\mathrm{Su}$ et al. 2009).

$\dagger$ Herschel is an ESA space observatory with science instruments provided by European-led Principal Investigator consortia and with important participation from NASA. 

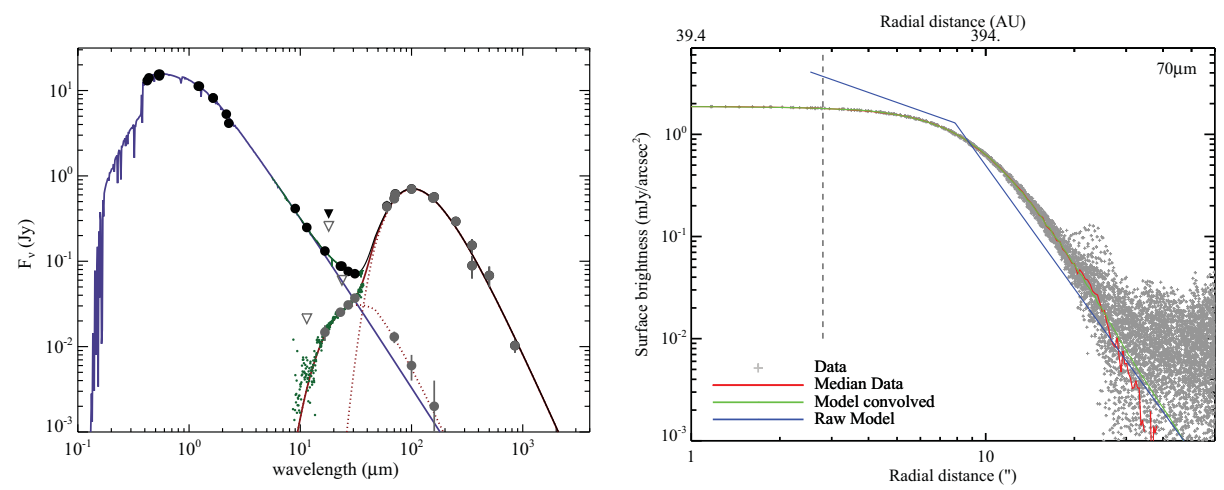

Figure 1. (left) The SED of the HR 8799 disk is shown, with independent curves for the warm blackbody component and the modified cold blackbody component. The stellar emission is fit with a $T_{\text {eff }}$ of $7380 \mathrm{~K}$ and $L_{*}=5.4 L_{\odot}$, consistent with a late-A or early F-type star. The temperatures of the disk components are $153 \pm 15 \mathrm{~K}$ (the warm unresolved component not detected by Herschel) and $36 \pm 1 \mathrm{~K}$ (the cold resolved component). There is no temperature distinction between the halo and the planetesimal belt evident in the SED. (right) The radial profile of the disk at $70 \mu \mathrm{m}$. The HWHP is indicated by a dashed line. The break in the surface brightness profile around $10^{\prime \prime}$ is apparent and justifies the interpretation of a two-component model for the outer disk. The data constrain the surface brightness profile very well out to $20-30^{\prime \prime}(800-1200 \mathrm{AU})$. Figures are from Matthews et al. (2013), in preparation.

The Herschel images (not shown here) do not exhibit any azimuthal asymmetries. The radial profile produced by azimuthally averaging the data is shown in Figure 1. From this profile, it is clear that the cold component of the disk is comprised of material with two different distributions. The planetesimal belt exhibits a shallow profile from $90-310 \mathrm{AU}$ (the inner radius is constrained to within $\pm 10 \mathrm{AU}$ ) while the halo exhibits a much steeper profile. Separate fits to the resolved images show that the index of the radial profile of the planetesimal belt is $\sim-1$ (constraint of this parameter is degenerate with the unresolved central flux and the unresolved inner edge of the belt) while the better constrained halo profile index has values of $-4.0 \pm 0.3$ and $-3.5 \pm 0.5$ at 70 and $100 \mu \mathrm{m}$ respectively. We are able to trace the halo emission to a radius of $2000 \mathrm{AU}$ from the central star. We discuss the non-standard filtering applied to reconstruct the maps to capture the largest scale structures in Matthews et al. (2013).

These data illustrate that structural features in disks can be masked by the modeling of SEDs in the absence of resolved imaging. The fact that the halo of HR 8799 matches so closely the temperature of the planetesimal belt suggests that the grains are almost blackbodies. Non-blackbody grains would necessarily emit less efficiently than blackbodies and would therefore exhibit higher temperatures to maintain thermal equilibium.

\section{References}

Griffin, M. J., Abergel, A., Abreu, A., et al. 2010, A\&A, 518, L3

Ishihara, D., Onaka, T., Kataza, H., et al. 2010, A\&A, 514, A1

Poglitsch, A., Waelkens, C., Geis, N., et al. 2010, A\&A, 518, L2

Matthews, B. C., et al. 2013, ApJ, in press

Marois, C., Macintosh, B., Barman, T., et al. 2008, Science, 322, 1348

Marois, C., et al. 2010, Nature, 468, 1080

Moshir, M., Kopan, G., Conrow, T., et al. 1990, Bull. AAS, 22, 1325

Patience, J., Bulger, J., King, R. R., et al. 2011, A\&A, 531, L17

Su, K. Y. L., Rieke, G. H., Stapelfeldt, K. R., et al. 2009, ApJ, 705, 314

Williams, J. P. \& Andrews, S. M. 2006, ApJ, 653, 1480 